\title{
Quantitative Microanalysis at Low Voltage with a WDS Electron Microprobe Equipped with a FE Column
}

\author{
Andrew N. Davis ${ }^{1}$, Chrystel Hombourger ${ }^{2}$ and Michel Outrequin ${ }^{2}$ \\ 1. CAMECA Instruments, Inc., 5500 Nobel Drive Suite 100, Madison, WI 53711-4951 \\ 2. CAMECA, 29 Quai des Grésillons, 92622 Gennevilliers Cedex, France
}

Thanks to its precision, its reproducibility and its stability, Electron Microprobe is a well suited technique for accurately analyze nearly all chemical element to concentration level down to few 10's ppm with a spatial resolution of about $1 \mu \mathrm{m}$, which is relevant to microstructures in a wide variety of materials and mineral specimens.

With the development of the Schottky emitter and its implementation as electron source in Electron Microprobe, small features are commonly analyzed own to sub-micrometer scale. Thanks to the high brightness of the Schottky emitter, fine focused electron beam can be achieved with both high and stable beam currents even low accelerating voltages $(\leq 10 \mathrm{keV})$.

Since X-rays are generated from a much larger diameter than the diameter of the incident electron beam, it is necessary to optimize the two interdependent parameters, accelerating voltage and beam diameter, in order to take full advantage of the FEG electron source for X-ray analysis. The electron beam diameter increases with decreasing the electron beam energy as shown on figure 1 . The interaction volume - within which scattered electrons generate X-rays - decreases with the electron energy as shown on Monte Carlo simulation run with the program CASINO [1] on figure 2 . Thus a small beam diameter is not always synonym of a small interaction volume and optimized conditions are obtained when the analytical spatial resolution is primarily limited to the diameter of the X-ray emission volume in a specific material.

The ability to accurately quantify precipitate phases on the micrometer and sub-micrometer scale when working at low beam energy with high spatial resolution will be illustrated in examples as Dunite, igneous rock locally enriched in Platinum acquired on the CAMECA SXFiveFE. The analytical resolution determined from X-ray maps will be presented.

\section{References:}

[1] D. Drouin, A. Réal Couture, D. Joly, X. Tastet, V. Aimez, R. Gauvin, Scanning, 29 (2007), 92-101.

[2] The authors acknowledge Ph. De Parseval et B. Abily from GET for sharing samples. 


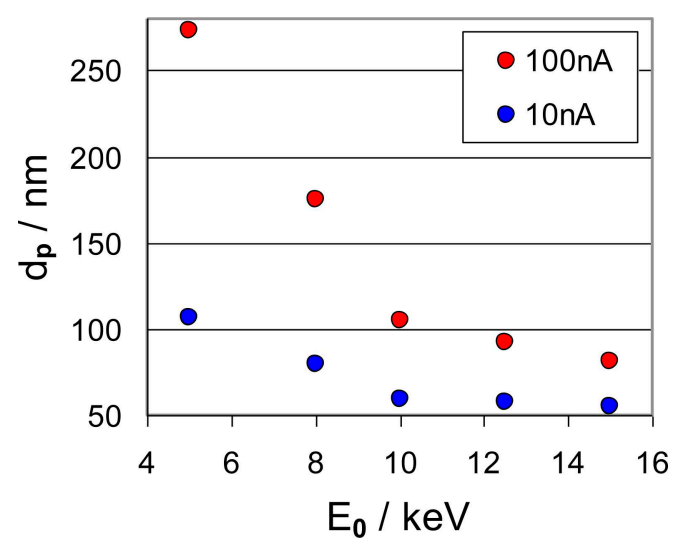

Figure 1: Beam diameter versus beam accelerating voltage at 2 beam currents (blue $=10 \mathrm{nA}$, red $=100 \mathrm{nA}$ ).

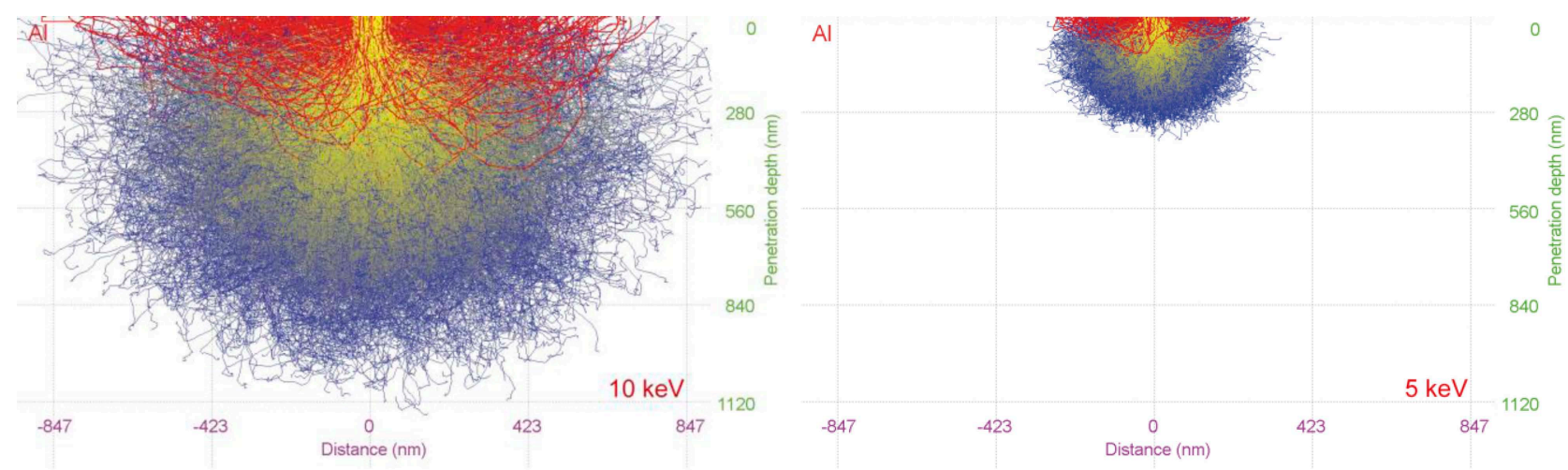

Figure 2: Monte Carlo simulations of electron trajectories beneath the specimen surface as calculated by CASINO [1]. Note the decrease in the interaction volume for $\mathrm{Al}$ as the beam energy decreases by about $60 \%$ from $10 \mathrm{keV}$ to $5 \mathrm{keV}$ (red = backscattered electrons, yellow = other electrons of high energy, blue $=$ electrons of low energy). 